\section{Skeletal Muscle Ultrasonography in Children with a Dysfunction in the Oxidative Phosphorylation System}

\author{
S. Pillen ${ }^{1,3}$ \\ E. Morava 2,3 \\ M. Van Keimpema ${ }^{1}$ \\ H. J. Ter Laak ${ }^{4}$ \\ M. C. De Vries 2,3 \\ R. J. Rodenburg ${ }^{2}$ \\ M. J. Zwarts ${ }^{1}$
}

\section{Abstract}

Objective: The aim of this prospective study was to investigate the diagnostic value of quantitative skeletal muscle ultrasonography in children suspected of having a mitochondrial disorder. Methods: Muscle thickness and quantitatively determined echo intensity of four muscles were established in 53 children with symptoms indicative of a mitochondrial disorder. Results: A sensitivity of 25 to $46 \%$ was found, depending on the chosen cut-off point (abnormal or borderline abnormal), with a specificity of 85 to $100 \%$. Except for one, all abnormal ultrasound scans were found in children over five years of age. Within the group of patients with a mitochondrial disorder, a significant correlation was found between muscle echo intensity and age $(r=0.38$; $\mathrm{p}=0.047$ ). Conclusions: We conclude that skeletal muscle ultrasound can be of additional value in the diagnosis of children with suspected mitochondrial disorders, especially in children over five years of age. With its low sensitivity, it is not suitable for screening purposes. However, since all abnormal ultrasound scans were found in children with a mitochondrial disorder, and no significant correlation with the MDC score was found, muscle ultrasound can be used complementary to this scoring system to facilitate the decision-making in pursuing further invasive diagnostics.

\section{Key words}

Mitochondrial disorders · muscle ultrasound $\cdot$ children · diagnostic value

\section{Introduction}

Oxidative phosphorylation (OXPHOS) is the major source of energy production of the cell, and takes place in the mitochondria. Mitochondrial disorders are the most common inborn errors of metabolism with an estimated frequency of 1:10000 live birth [23]. Due to the central role of mitochondria in energy metabolism, mitochondrial diseases are often multi-organ disorders with predominant involvement of brain and muscles [25]. To diagnose defects in OXPHOS, muscle is the tissue of choice to be examined. As the collection of a muscle specimen requires an invasive procedure, it would be desirable to have a diagnostic instrument to determine if these invasive investigations are required.

Previous investigations have demonstrated that some patients with mitochondrial myopathies showed marked atrophy and fatty infiltration on CT or MRI, which was correlated with an increased fatty infiltration in the muscle biopsy $[5,14,18,31]$. However, these investigations have mainly been limited to adults with specific mitochondrial DNA mutations.

In children, imaging studies of skeletal muscles have predominantly been performed with ultrasonography because of its non-invasive nature and the avoidance of sedation. Neuromuscular disorders generally cause increased muscle echo intensity, which correlates positively with the infiltration of fat and fibrous tissue $[8,22]$. Although this technique has proven to be a very

Affiliation

${ }^{1}$ Department of Clinical Neurophysiology, Institute of Neurology, Radboud University Nijmegen

Medical Centre, Nijmegen, The Netherlands

${ }^{2}$ Nijmegen Centre for Mitochondrial Disorders, Radboud University Nijmegen

Medical Centre, Nijmegen, The Netherlands

${ }^{3}$ Department of Paediatrics, Radboud University Nijmegen Medical Centre, Nijmegen, The Netherlands

${ }^{4}$ Department of Pathology and Neurology, Radboud University Nijmegen Medical Centre, Nijmegen, The Netherlands

Correspondence

Dr. Sigrid Pillen · Department of Clinical Neurophysiology · Department of Paediatrics · Radboud University Nijmegen Medical Centre·P.O. Box 9101 · 6500 HB Nijmegen·Netherlands·E-mail: s.pillen@cukz.umcn.nl

Received: June 12, 2006 · Accepted after Revision: July 10, 2006 
sensitive method to detect structural changes in muscles caused by neuromuscular disorders in general $[9,19,32]$, lower sensitivities can be expected in the subgroup of mitochondrial myopathies, since children with mitochondrial myopathies rarely show structural changes in muscle biopsy. However, up to 70 percent of these patients did show an abnormal ultrasound scan, suggesting that muscle ultrasonography could also be helpful in the diagnosis of these patients $[3,9,19]$. One could hypothesize that in these patients, the shortage of energy production of the muscle could lead to metabolic stress, resulting in muscle damage, which would be visible on muscle ultrasound, as it is in adults on other image modalities. But until now, the diagnostic value of skeletal muscle ultrasonography in children with suspected mitochondrial disorders has never been systematically investigated.

The purpose of this prospective study was therefore to investigate the diagnostic value of skeletal muscle ultrasound in children with suspected mitochondrial disorders. We hypothesized that its sensitivity is correlated to the age of the child and the type and severity of the mitochondrial disorder: Older age and more severe biochemical abnormalities might be correlated with a higher number of structural changes in the muscle $[13,16]$. We therefore correlated our findings to the age of the patient, type of mitochondrial disorder, severity of symptoms and biochemical abnormalities.

\section{Methods}

\section{Patients}

Between May 2004 and September 2005, we prospectively included 53 children referred to our tertiary centre for metabolic disorders, because of a suspected mitochondrial disorder. The study was approved by the local ethics committee, and informed consent was obtained of all parents and patients when older than 12 years of age. As part of the regular work-up in children with a suspected disorder in the oxidative phosphorylation we analysed the blood lactate (multiple measurements) and pyruvate levels, blood gas, serum acyl-carnitine, serum and urine amino acid profile, urine organic acid profiles. Cerebral spinal fluid investigations were performed depending on the presence of central nervous system involvement. Furthermore, the patients underwent a diagnostic protocol of multiple investigations including ECG, chest X-ray, EEG, visual evoked potentials, acoustic evoked potentials, sensory evoked potentials and a cerebral MRI. Muscle ultrasonography was performed in this initial evaluation phase of the diagnostics.

Based on a previous prospective clinical and biochemical study a consensus mitochondrial disease criteria (MDC) scoring system has been established to facilitate the diagnosis in patients with a suspected mitochondrial disorder. The MDC score is positively correlated to the likelihood of a mitochondrial disorder, confirmed by the biochemical abnormalities measured in a fresh muscle biopsy [30]. The MDC system uses a combined scoring of muscle symptoms (maximal score: 2 points), CNS abnormalities (maximal score: 2 points) and multi-system involvement (maximal score: 3 points) with a maximal total clinical score of 4 points, and the addition of metabolic abnormalities and the re- sults of the neuroimaging (maximal additional score: 4 points). Specific histologic anomalies found at the time of the biopsy might increase the final MDC score further with 4 points, leading to a total maximal score of 12 points [30]. The scoring system was originally established as a method to achieve the final clinical diagnosis. In case of a high clinical/metabolic section of the MDC score, the application of the criteria facilitates the decision making in the further diagnostic steps already prior to the muscle biopsy. Conversely, a low MDC score makes the presence of mitochondrial dysfunction very unlikely. In the present study we used the MDC score to clinically evaluate mitochondrial dysfunction. If a patient had a MDC score lower than 3 or improving clinical signs, a muscle biopsy was not performed. If a patient had a MDC score of 3 points or higher we performed a muscle biopsy to confirm the diagnosis on the biochemical level.

\section{Muscle biopsy and biochemical analysis}

After the initial evaluation, the patient's clinician decided whether an open muscle biopsy was indicated, based on the MDC score (3 or higher), the presence of persistent and/or progressive symptoms and the eventual familial occurrence of the symptoms. Muscle biopsy was performed under general anaesthesia within three months. In children with a spontaneously improving clinical picture and decreasing MDC scores the biopsy was postponed and further follow-up was initiated. Due to the prospective nature of the study, both groups of patients (with or without a muscle biopsy) were included for further analysis.

A part of each muscle biopsy was used for histopathological examination. Frozen muscle sections were stained according to standard histological and enzyme histochemical methods and were evaluated for evidence of mitochondrial disorders (COX negative or COX deficient fibres and ragged-red fibres) and other neuromuscular disorders. Since structural abnormalities like fatty infiltration and fibrosis may correlate with abnormal muscle ultrasonography, percentage of fat cells and percentage perimysial tissue (for the larger part containing connective tissue, but also blood vessels and nerve bundles may be present) were measured for each muscle biopsy. Pictures from Sudan Black B stained sections served to determine the fat cell area. Trichrome stained sections were used for perimysial tissue measurements. For both measurements a systematic random point sampling design was used. The results were obtained by summing the points covering the fat cell area or connective tissue area in relation to the respective total area of the biopsy section.

Detailed biochemical investigations, including substrate oxidation rates (pyruvate, malate, succinate), ATP production rate from pyruvate oxidation and the activity of the mitochondrial complexes I, II, III, IV, V and PDHc were measured in a fresh muscle sample according to previously described methods $[10,11,29]$.

\section{Molecular genetic analysis}

Mutation analysis of the MELAS 3243A>G, MERRF 8344A $>G$ and Leigh/NARP 8993T $>C$, sequence analysis of the mtDNA for mutations in the genes ND1, ND2, ND3, ND4, tRNA Leu (uur) and tRNA Lys, long template PCR analysis for mitochondrial deletions and sequence analysis of the nuclear encoded PDHA1, SURF1, NDUSF1, NDUSF2, NDUSF4, NDUSF7 and NDUVF1 genes were performed in 
the patients diagnosed with a definite mitochondrial disorder, appropriate to the clinical presentation and the detected biochemical alterations.

\section{Gold standard}

Since the underlying mutation is only discovered in the minority of pediatric patients with mitochondrial disorders $[23,26]$, we applied a functional-biochemical definition to validate muscle ultrasonography. Based on biochemical evaluation and muscle biopsy, the children were assigned to the following groups:

1. Mitochondrial disorder:

a) definite: decreased pyruvate oxidation rate, ATP production rate, and at least one enzyme complex activity below the control range

b) possible: decreased pyruvate oxidation rate, ATP production rate below the control range but normal enzyme complex activities

2. No mitochondrial dysfunction:

a) excluded on the biochemical level (with muscle biopsy)

b) no mitochondrial dysfunction suspected/no biopsy requested: low MDC score, improvement of clinical signs.

3. Other diagnosis.

\section{Skeletal muscle ultrasound measurement}

In all patients an ultrasound measurement was obtained before the diagnosis had been established and before a muscle biopsy had been performed. The investigator was blinded for the clinical presentation and the results of other investigations. The method of ultrasound scanning is described in detail in a previous study of our group [24]. As the ultrasound measurement might be applied as a screening tool, a short protocol was chosen, in which four muscles were selected for examination: two proximal, two distal, in both arms and legs (biceps brachii, forearm flexors, quadriceps femoris, and tibialis anterior muscle). With this protocol, all measurements could be performed within 15 minutes.

All muscles were examined in the transverse plane. A phased array real-time scanner (Sonos 2000 Phased Array Imaging System; Hewlett-Packard Company, Andover, Massachusetts, USA) was used, with a $7.5 \mathrm{MHz}$ transducer. All system-setting parameters were conform our previous study, to be able to use the established normal values (gain $86 \mathrm{~dB}$, compression $70 \mathrm{~dB}$, no adjustments in time gain compensation or focus). The images were digitized and stored as "tagged image file format" (TIFF) files with a resolution of $800 \times 600$ pixels with 256 grey-levels. Three consecutive measurements were taken of every muscle and results were averaged. After each measurement the patient was allowed to move and the transducer was repositioned. During the examination of the muscles, care was taken to maintain the same standardized position of the child and the exact location of the transducer as described in our previous study with healthy children [24]. Muscle thickness was measured with electronic callipers during the examination. Echo-intensity (EI) was determined using computer-assisted grey-scale analysis. In each image, the entire muscle was selected just within the borders of the muscle. In the quadriceps muscle, the rectus femoris muscle was chosen for analysis of EI, as in severe neuromuscular disorder the outlines of the vastus intermedius muscle can be difficult to define [7]. The mean EI of this region was then calculated us- ing the standard histogram function of Adobe Photoshop (Adobe Systems Inc., San Jose, California, USA).

As muscle echo intensity is different for each muscle and muscle thickness is dependent on weight [24], it has to be standardized to be able to compare individual patients and muscles. The measurements were therefore compared with normal values, established in a previous study [24], and transformed into z-scores (expressed in standard deviations from normal, SD), using the following equation:

$\mathrm{Z}-$ score $(\mathrm{SD})=\frac{\text { measured value }- \text { normal value }}{\text { standard deviation of normal value }}$

\section{Statistical analysis}

Our data were analyzed with the SPSS package for Windows 12.0.1 (SPSS Inc., Chicago, Illinois, USA) and statistical significance was defined as $p$ value $\leq 0.05$. The standardized echo intensity and muscle thickness were compared to normal using a one-sample $t$-test, and compared between patients with a mitochondrial disorder (definite and possible) and patients without a mitochondrial disorder using an independent samples MannWhitney U test.

Sensitivity and specificity were determined after applying two cut-off points:

- An abnormal ultrasound measurement was defined as one very abnormal muscle (z-score $>3.5 \mathrm{SD}$ ) or 2 abnormal muscles (> 2.5 SD) or 3 slightly abnormal muscles (> 1.5 SD).

- A normal ultrasound measurement was defined as all muscles below 2.0 SD and three muscles below 1.5 SD and two muscles below 1.0 SD.

- Between these cut-off points, the ultrasound measurement was called borderline abnormal.

These cut-off points were chosen because, in this way, both patients with selective muscle involvement and patients with generalized muscle involvement would be detected with the highest predictive values.

To determine the cause of increased echo intensity in patients with mitochondrial disorders, the sum of all muscles were correlated to age, initial MDC-score, ATP production, and percentage fat and fibrous tissue in the muscle biopsy. Two combined terms were added because of the hypothesis that both age and severity of the disorder would determine the amount of muscle damage: MDC $\times$ age and ATP/age. The correlations were determined with a Pearson's correlation coefficient.

\section{Results}

Forty-eight children were assigned to the diagnostic groups as follows: Twenty-eight children had a definite or possible mitochondrial disorder; seventeen children did not show any evidence of a mitochondrial disorder. In the group of the children with a definite mitochondrial disorder one patient was diagnosed with a common ATPase 6 mtDNA mutation, and another one with a nuclear-encoded homozygous SURF1 mutation. The three remaining patients were diagnosed with Prader-Willi syndrome $(n=1)$ or a chromosome disorder with secondary mito- 
Table 1 Diagnosis, age, MDC score, ATP production and ultrasound results of all patients

\begin{tabular}{|c|c|c|c|c|c|}
\hline & \multicolumn{2}{|c|}{ Mitochondrial disorder } & \multicolumn{2}{|c|}{ No mitochondrial disorder } & Other diagnosis" \\
\hline Number of patients & 13 & 15 & 7 & 10 & 3 \\
\hline $\begin{array}{l}\text { US results (abnormal/ } \\
\text { borderline abnormal [n]) }\end{array}$ & $3 / 2$ & $4 / 4$ & $0 / 1$ & $0 / 2$ & $0 / 0$ \\
\hline MDC score (median, range) & $6(3-11)$ & $6(3-8)$ & $4(1-6)$ & $4.5(2-6)$ & $2 ; 2 ; 6$ \\
\hline Number of patients with biopsy & 13 & 15 & 7 & 0 & 2 \\
\hline ATP production (mean, range) & $25(6-43)$ & $32(21-40)$ & $48(43-70)$ & ND & $27 ; 34$ \\
\hline Biopsy \% fat (median, range) & $0.6(0-10)$ & $0.4(0-7.0)$ & $1.9(0-4.2)$ & ND & $0.0 ; 0.4$ \\
\hline $\begin{array}{l}\text { Biopsy \% fibrous tissue } \\
\text { (median, range) }\end{array}$ & $2.6(0.4-6.7)$ & $1.0(0-6.5)$ & $3.2(1.1-14)$ & ND & $0.9 ; 3.3$ \\
\hline
\end{tabular}

* Prader-Willi syndrome, chromosomal disorder with secondary mitochondrial dysfunction $(n=2)$. ND = not determined; US = ultrasound

chondrial dysfunction $(\mathrm{n}=2)$. Table 1 summarizes the results of the diagnostic evaluation. Five children were excluded from further analysis: one child was lost to follow-up; muscle biopsy was indicated but postponed in four children due to a significant spontaneous improvement or social problems.

Muscle thickness of all muscles, except for the forearm flexors, was significantly decreased in all patients, with or without a mitochondrial disorder, but was not significantly different between patients with and without a mitochondrial disorder. Muscle echo intensity was significantly higher in all muscles, except for the forearm flexors, in patients with a mitochondrial disorder compared to patients without a mitochondrial disorder (Table 2). Fig. 1 shows an example of two ultrasound scans of the quadriceps.

Seven out of 28 children with a definite or possible mitochondrial disorder had an abnormal ultrasound scan. With the exception of one child, all these children were over five years of age. Six patients had a borderline abnormal ultrasound scan. This gives a sensitivity of 25 to $46 \%$ depending on the cut-off point chosen (abnormal or borderline abnormal). No child without a mitochondrial disorder or other diagnosis had an abnormal ultrasound scan; three had a borderline abnormal ultrasound scan. This gives a specificity of 85 to $100 \%$.

Within the group of patients with a possible or definite mitochondrial disorder, a significant correlation was found between the sum of muscle echo intensity of all muscles and age $(r=0.38 ; p=0.047$; Fig. 2$)$. The MDC-score, ATP production, the combination of these parameters with age did not correlate significantly with muscle echo intensity. Both intramuscular fat percentage $(\mathrm{r}=0.34 ; \mathrm{p}=0.079)$ and intramuscular connective tissue $(\mathrm{r}=-0.038 ; \mathrm{p}=0.85)$ were not significantly correlated with muscle echo intensity of the quadriceps muscle.
Table 2 Differences in muscle thickness and echo intensity between patients with and without a mitochondrial disorder

\begin{tabular}{|c|c|c|c|}
\hline & $\begin{array}{l}\text { Mitochondrial } \\
\text { disorder } \\
\text { (z-score) }\end{array}$ & $\begin{array}{l}\text { No mitochon- } \\
\text { drial disorder } \\
\text { (z-score) }\end{array}$ & $\begin{array}{l}\text { Significance } \\
\text { of difference } \\
\text { (p-value) }\end{array}$ \\
\hline \multicolumn{4}{|l|}{ Muscle thickness } \\
\hline Biceps brachii & $-0.95^{*}$ & $-1.1^{*}$ & n.s. \\
\hline Forearm flexors & -0.10 & 0.06 & n.s. \\
\hline Quadriceps femoris & $-1.36^{*}$ & $-1.33^{*}$ & n.s. \\
\hline Tibialis anterior & $-0.44 *$ & $-0.63 *$ & n.s. \\
\hline \multicolumn{4}{|l|}{ Echo intensity } \\
\hline Biceps brachii & $0.39^{\dagger}$ & -0.63 & 0.001 \\
\hline Forearm flexors & 0.30 & -0.16 & n.s. \\
\hline Quadriceps femoris & $1.46^{\dagger}$ & $0.50^{\dagger}$ & 0.010 \\
\hline Tibialis anterior & $1.10^{\dagger}$ & 0.13 & 0.001 \\
\hline
\end{tabular}

* significantly decreased compared to normal, ${ }^{\dagger}$ significantly increased compared to normal

\section{Discussion}

In this prospective study, we investigated the value of muscle ultrasonography in the diagnostic work-up in children with a suspected dysfunction in the OXPHOS. We found a sensitivity of up to $46 \%$, which was lower than the sensitivity reported in children with other neuromuscular disorders [9,32]. With this sensitivity, muscle ultrasound is not suitable for screening purposes since a negative ultrasound result will not exclude a mitochondrial myopathy [2]. However, muscle ultrasound did show a very high positive predictive value: all abnormal ultrasound results were found in children with a "definite" or "possible" mitochondrial disorder. The ultrasound technique could therefore be used in addition to other investigations and the clinical diagnostic scoring system. In children with a clinically uncertain diagnostic score, a positive ultrasound would support the decision to perform further, more invasive investigations (e.g. muscle biopsy). 


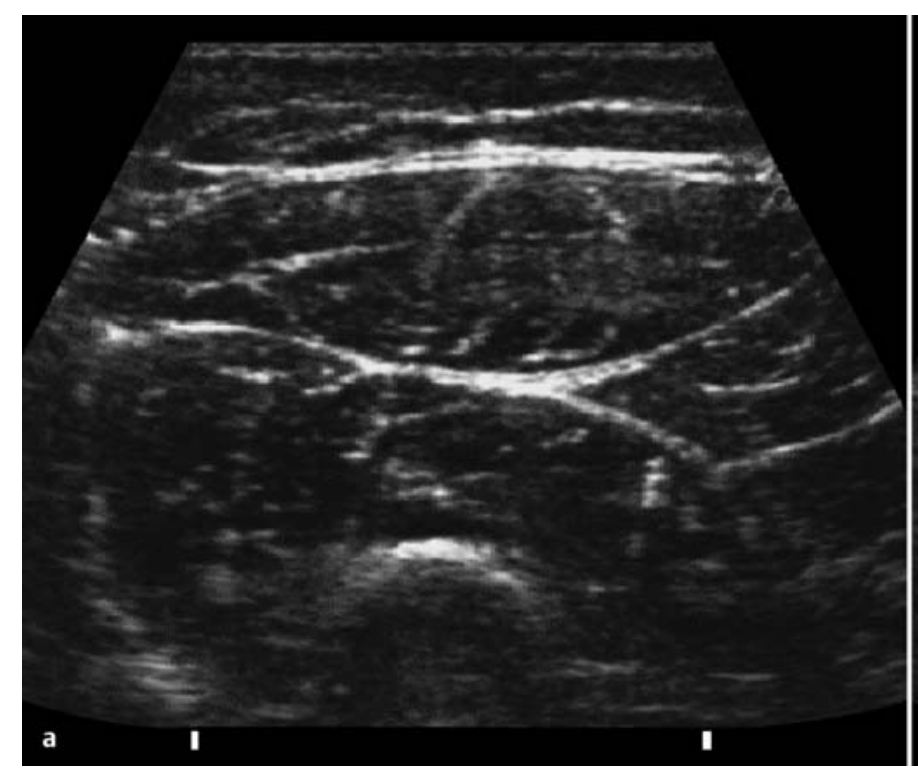

Fig. $1 \mathbf{a}$ and $\mathbf{b}$ Transverse ultrasound scan of the quadriceps muscle. a normal muscle appearance of a 6-year-old patient without a mitochondrial disorder. $\mathbf{b}$ abnormal ultrasound scan with increased echo

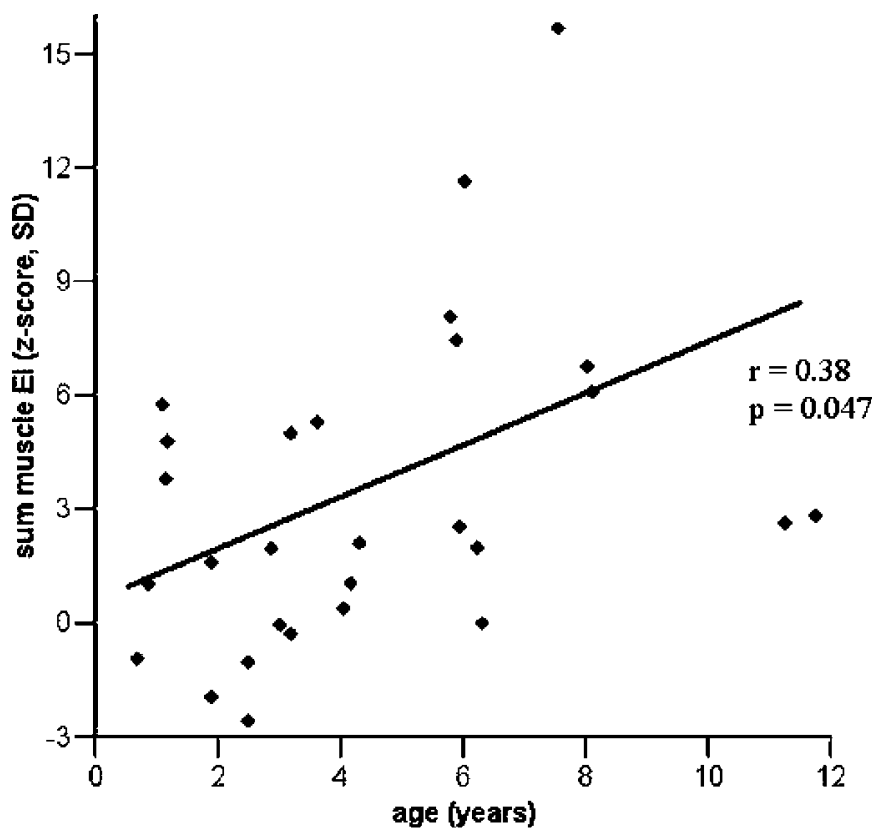

Fig. 2 Correlation between age and muscle echo intensity in patients with a mitochondrial disorder $(n=28)$.

In our study group, almost all abnormal ultrasound results were found in children over five years old, indicating that the diagnostic value is higher in older children. Conversely, as the sensitivity is very low in younger children, skeletal muscle ultrasonography is not very likely to contribute to the diagnosis in young children with mitochondrial disorders. Moreover, based on these results one could state that if an abnormal ultrasound result is found in a child younger than 5 years of age, the presence of another neuromuscular disorder might be more likely $[9,16]$.

Ultrasonography is a non-invasive and easily available technique and suitable for use in children. Other imaging modalities have also been used in the diagnosis of neuromuscular disorders in

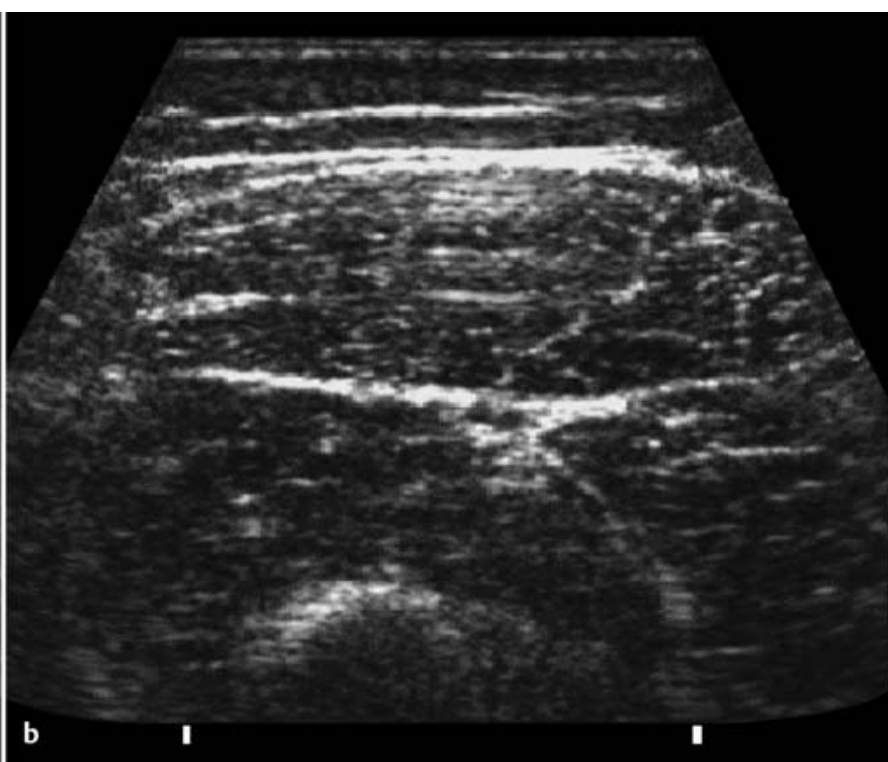

intensity of the rectus femoris muscle. This patient, who was also six years old, suffered from a complex I, III and V deficiency.

children. The use of muscle MRI has been investigated extensively in children with neuromuscular disorders $[6,15,21]$, but, although no prospective studies have been performed, it seems to be less capable of detecting pathological changes in children with mitochondrial myopathies [1,4]. Moreover, the major disadvantages of MRI are high costs and the prerequisite of sedation in young or mentally retarded children.

A significant correlation between age and echo intensity was found in the group of children with mitochondrial dysfunction, where older children showed higher muscle echo intensities. This is in concordance with previous investigations, in which higher sensitivities in adults and increased alterations with age were reported $[5,14,18,20]$. The defect in OXPHOS results in metabolic stress during exercise, which can cause damage to DNA and apoptosis [16], eventually leading to structural changes like fibrosis and fatty infiltration, accumulating over years. This phenomenon may explain the increase in severity of the disease with age [13]. However, the prospective nature of the study resulted in an inclusion of younger children with relatively severe and older children with a relatively mild presentation of a mitochondrial disorder. This probably explains the limited correlation with age found in this study. Another study including children with various duration of their illness or a longitudinal study design would be more appropriate to investigate if an increase in echo intensity occurs when children with mitochondrial disorders grow older.

The absence of a significant correlation between the MDC score and the muscle echo intensity indicates that the presence of clinical symptoms and muscle involvement does not lead directly to increased echo intensity, and that muscle ultrasound can therefore be used complementary to this diagnostic scoring system. Previous studies have shown that muscle echo intensity is strongly correlated with structural changes in the muscle biopsy, especially fatty infiltration. However, in the present study the correlation between percentage intramuscular fat and echo in- 
tensity was not very strong, probably because only a few patients showed a pathologically increased percentage of fat. Moreover, the location and size of fat collections also effects echo intensity. Structures smaller than the wavelength of the ultrasound beam do not cause a reflection and do therefore not increase echo intensity $[3,8]$. This fact was not taken into account in the correlation calculated.

In this study, the diagnosis of most patients was based on the clinical evaluation and confirmed with biochemical measurements in a fresh muscle biopsy, which gives a sensitive, functional information about mitochondrial function. Since an underlying gene mutation is only discovered in the minority of pediatric patients with mitochondrial disorders, we applied this functional-biochemical definition to validate muscle ultrasonography. In many adult patients, especially in the familial cases, mtDNA-defects could be confirmed early on in the diagnostic phase. In children however, most OXPHOS disorders are caused by de novo nuclear gene defects, most of which still await identification $[23,26,28]$. However, mitochondrial function can be temporarily or secondarily impaired due to extreme inactivity, malnutrition or other neuromuscular disorders $[12,17,27]$. On the other hand, biochemical results can be normal, even in patients with a definite disorder in OXPHOS and a known mitochondrial DNA mutation, for example if the percentage of heteroplasmy in the muscle tissue under examination is below the threshold level that gives rise to a detectable OXPHOS defect. This has to be taken into account when interpreting the results of our study, but could not be avoided because of its prospective design.

We conclude that skeletal muscle ultrasound can be of additional value in the diagnosis of children between 5 and 18 years of age with suspected mitochondrial disorders. With its low sensitivity, it is not suitable for screening purposes. However, since a high positive predictive value was found and no significant correlation with the MDC score, it could be used complementary to this scoring system to facilitate the decision-making in requesting further invasive diagnostics.

\section{References}

${ }^{1}$ Chan WP, Liu GC. MR imaging of primary skeletal muscle diseases in children. AJR Am J Roentgenol 2002; 179: 989-997

${ }^{2}$ Feinstein AR. Clinical Epidemiology; the Architecture of Clinical Research. Philadelphia: W. B. Sauders Company, 1985: 597-631

${ }^{3}$ Fischer AQ Carpenter DW, Hartlage PL, Carroll JE, Stephens S. Muscle imaging in neuromuscular disease using computerized real-time sonography. Muscle Nerve 1988; 11: 270-275

${ }^{4}$ Fleckenstein JL. MRI of neuromuscular disease: the basics. Semin Musculoskelet Radiol 2000; 4: $393-419$

${ }^{5}$ Fleckenstein JL, Haller RG, Girson MS, Peshock RM. Focal muscle lesion in mitochondrial myopathy: MR imaging evaluation. J Magn Reson Imaging 1992; 2: 121

${ }^{6}$ Fleckenstein JL, Reimers CD. Inflammatory myopathies: radiologic evaluation. Radiol Clin North Am 1996; 34: 427-439

7 Heckmatt JZ, Dubowitz V, Leeman S. Detection of pathological change in dystrophic muscle with B-scan ultrasound imaging. Lancet 1980; 1: $1389-1390$

${ }^{8}$ Heckmatt JZ, Leeman S, Dubowitz V. Ultrasound imaging in the diagnosis of muscle disease. J Pediatr 1982; 101: 656-660
${ }^{9}$ Heckmatt JZ, Pier N, Dubowitz V. Real-time ultrasound imaging of muscles. Muscle Nerve 1988; 11: 56-65

${ }^{10}$ Janssen AJ, Smeitink JA, van den Heuvel LP. Some practical aspects of providing a diagnostic service for respiratory chain defects. Ann Clin Biochem 2003; 40: 3-8

11 Janssen AJ, Trijbels FJ, Sengers RC, Wintjes LT, Ruitenbeek W, Smeitink JA et al. Measurement of the energy-generating capacity of human muscle mitochondria: diagnostic procedure and application to human pathology. Clin Chem 2006; 52: 860-871

12 Jongpiputvanich S, Sueblinvong T, Norapucsunton T. Mitochondrial respiratory chain dysfunction in various neuromuscular diseases. J Clin Neurosci 2005; 12: 426-428

${ }^{13}$ Kang D, Hamasaki N. Alterations of mitochondrial DNA in common diseases and disease states: aging, neurodegeneration, heart failure, diabetes, and cancer. Curr Med Chem 2005; 12: 429-441

${ }^{14}$ Karppa M, Mahjneh I, Karttunen A, Tolonen U, Majamaa K. Muscle computed tomography patterns in patients with the mitochondrial DNA mutation 3243A > G. J Neurol 2004; 251: 556-563

${ }^{15}$ Lovitt S, Marden FA, Gundogdu B, Ostrowski ML. MRI in myopathy. [Review]. Neurol Clin 2004; 22: 509-538

${ }^{16}$ Mitsui T, Kawai H, Nagasawa M, Kunishige M, Akaike M, Kimura Y et al. Oxidative damage to skeletal muscle DNA from patients with mitochondrial encephalomyopathies. J Neurol Sci 1996; 139: 111 - 116

17 Morava E, Dinopoulos A, Kroes HY, Rodenburg RJ, van Bokhoven $\mathrm{H}$ van den Heuvel LP et al. Mitochondrial dysfunction in a patient with Joubert syndrome. Neuropediatrics 2005; 36: $214-217$

18 Olsen DB, Langkilde AR, Orngreen MC, Rostrup E, Schwartz M, Vissing $\mathrm{J}$. Muscle structural changes in mitochondrial myopathy relate to genotype. J Neurol 2003; 250: 1328 - 1334

${ }^{19}$ Pillen S, Scholten RR, Zwarts MJ, Verrips A. Quantitative skeletal muscle ultrasonography in children with suspected neuromuscular disease. Muscle Nerve 2003; 27: 699-705

20 Reichmann H, Gold R, Meurers B, Naumann M, Seibel P, Walter U et al. Progression of myopathology in Kearns-Sayre syndrome: a morphological follow-up study. Acta Neuropathol (Berl) 1993; 85: 679-681

${ }^{21}$ Reimers CD, Finkenstaedt M. Muscle imaging in inflammatory myopathies. Curr Opin Rheumatol 1997; 9: 475-485

${ }^{22}$ Reimers K, Reimers CD, Wagner S, Paetzke I, Pongratz DE. Skeletal muscle sonography: a correlative study of echogenicity and morphology. J Ultrasound Med 1993; 12: 73-77

23 Schaefer AM, Taylor RW, Turnbull DM, Chinnery PF. The epidemiology of mitochondrial disorders-past, present and future. Biochim Biophys Acta 2004; 1659: 115-120

24 Scholten RR, Pillen S, Verrips A, Zwarts MJ. Quantitative ultrasonography of skeletal muscles in children: normal values. Muscle Nerve 2003; 27: 693-698

25 Smeitink J, van den HL, DiMauro S. The genetics and pathology of oxidative phosphorylation. Nat Rev Genet 2001; 2: 342 - 352

26 Thorburn DR. Mitochondrial disorders: prevalence, myths and advances. J Inherit Metab Dis 2004; 27: 349-362

27 Ushmorov A, Hack V, Droge W. Differential reconstitution of mitochondrial respiratory chain activity and plasma redox state by cysteine and ornithine in a model of cancer cachexia. Cancer Res 1999; 59: $3527-3534$

${ }^{28}$ Uusimaa J, Remes AM, Rantala H, Vainionpaa L, Herva R, Vuopala K et al. Childhood encephalopathies and myopathies: a prospective study in a defined population to assess the frequency of mitochondrial disorders. Pediatrics 2000; 105: 598-603

${ }^{29}$ Wijnhoven JH, Janssen AJ, van Kuppevelt TH, Rodenburg RJ, Dekhuijzen PN. Metabolic capacity of the diaphragm in patients with COPD. Respir Med 2006; 100: 1064 - 1071

${ }^{30}$ Wolf NI, Smeitink JA. Mitochondrial disorders: a proposal for consensus diagnostic criteria in infants and children. Neurology 2002; 59: $1402-1405$

${ }^{31}$ Wortmann RL, DiMauro S. Differentiating idiopathic inflammatory myopathies from metabolic myopathies. Rheum Dis Clin North Am 2002; $28: 759-778$

32 Zuberi SM, Matta N, Nawaz S, Stephenson JB, McWilliam RC, Hollman A. Muscle ultrasound in the assessment of suspected neuromuscular disease in childhood. Neuromuscul Disord 1999; 9: 203-207 